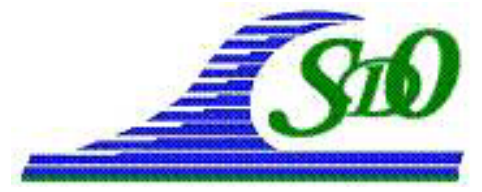

XI ìmes Journées Nationales Génie Côtier - Génie Civil

Les Sables d'Olonne, 22-25 juin 2010

DOI:10.5150/jngcgc.2010.040-H C Editions Paralia CFL

disponible en ligne - http://www.paralia.fr - available online

\title{
Étude numérique de la stabilité de la crème de vase en écoulement cisaillé
}

\author{
Alice HARANG ${ }^{1,2}$, Olivier THUAL ${ }^{1,2}$ \\ 1. Université de Toulouse, INPT, UPS, IMFT, Allée Camille Soula, \\ F-31400 Toulouse, France. \\ 2. CNRS, IMFT, F-31400 Toulouse, France. \\ harang@imft.fr ; thual@imft.fr
}

\section{Résumé :}

Afin d'améliorer la compréhension de la mise en suspension de la vase au fond des estuaires, une étude paramétrique de la stabilité de l'interface entre la crème de vase et l'eau est effectuée à l'aide d'un modèle bidimensionnel cisaillé de deux couches de fluides miscibles de masse volumique et de viscosité dynamique différentes. Le code de simulation numérique directe JADIM de l'IMFT est utilisé pour calculer l'évolution temporelle de ces écoulements. Le modèle est initialisé à l'aide de profils continus de type $\operatorname{erf}(z)$ pour toutes les grandeurs considérées. Cette étude de stabilité est de nature à déboucher sur de nouvelles paramétrisations pour la modélisation réaliste des estuaires.

\section{Mots-clés :}

Estuaire - Bouchon vaseux - Mélange - Stratification - Crème de vase - Mise en suspension - Transport sédimentaire

\begin{abstract}
:
To improve understanding of mud suspension in estuaries, a parametric stability analysis of the fluid-mud - water interface is performed with a two-dimensional shear flow of two miscible layers of different mass density and dynamical viscosity. The direct numeric simulation code JADIM of IMFT is used to compute the temporal evolution of these flows. The model is initialized with continuous $\operatorname{erf}(z)$ profiles for all quantities. Likely outcomes of this stability study are new parametrizations for realistic modelling of estuaries.
\end{abstract}

\section{Keywords:}

Estuary - Turbidity maximum - Mixing - Stratification - Mud flow - Entrainment Sediment transport

\section{Introduction}

Les modèles numériques des estuaires tels que, par exemple, TELEMAC_3D ou SIAM3D sont utilisés pour de nombreuses applications comme la gestion de la pollution ou les aménagements côtiers. Pour ce qui concerne la mise en suspension au niveau du fond de l'estuaire, ces modèles peuvent être améliorés par une étude plus approfondie de 
l'interface au niveau de la crème de vase comme le propose LE NORMAND (1995) avec le code TELEMAC_3D. Dans cet esprit, nous proposons ici une étude bidimensionnelle de la stabilité de cette interface. La stabilité et le mélange d'écoulements cisaillés et stratifiés a été étudié par CAULFIELD \& PELTIER (2000). ERN et al. (2003) ont étudié les écoulements cisaillés et stratifiés en viscosité. KRANENBURG \& WINTERWERP (1997) proposent un modèle validé expérimentalement, de mise en suspension de la crème de vase sous forçage turbulent de type houle ou vent. A notre connaissance, aucune étude ne s'est intéressée à des mélanges de type crème de vase, stratifiés en masse volumique et en viscosité, résultant du développement d'une instabilité de cisaillement.

\section{Méthodologie}

\subsection{Code de calcul JADIM}

Cette étude est menée avec le code de simulations numériques directes JADIM (LEGENDRE, 1996 ; HALLEZ, 2007 ; ERN et al., 2003). Ce code, sous la forme utilisée, résout le système d'équations :

$$
\begin{aligned}
& \nabla \cdot \underline{u}=0, \quad \frac{d \underline{u}}{d t}=-\frac{1}{\rho} \nabla P+\underline{g}+\frac{1}{\rho} \nabla\left[\mu\left(\nabla \underline{u}+{ }^{t} \nabla \underline{u}\right)\right], \\
& \frac{\partial C}{\partial t}+\nabla \cdot(C \underline{u})=0, \\
& \rho=\rho_{0}(1-C)+\rho_{1} C, \quad \mu=\mu_{0}(1-C)+\mu_{1} C, \quad \text { Erratum: }
\end{aligned}
$$

qui couple les équations de Navier-Stokes incompressibles (1.a), où $g$ est le vecteur gravité, à l'équation de transport (1.b) d'une fraction volumique $C$ qui contrôle, à travers les relations (1.c), le mélange cinématique de particules fluides de masse volumique $\rho$ et de viscosité dynamique $\mu$ inhomogènes. La diffusion moléculaire des sédiments est négligée (voir DEARDORFF \& WILLIS, 1982)

Nous considérons le mélange des deux fluides suivants : l'eau, de masse volumique $\rho_{0}=1000 \mathrm{~kg} \mathrm{~m}^{-3}$ et de viscosité dynamique $\mu_{0}=10^{-3} \mathrm{~Pa} \mathrm{~s}$, et la crème de vase, de masse volumique $\rho_{1}=1360 \mathrm{~kg} \mathrm{~m}^{-3}$ et de viscosité dynamique $\mu_{1}=1 \mathrm{~Pa}$. Ces deux entités sont ensuite mélangées et suivies par le taux de présence de crème de vase dans le fluide, $C$. Ainsi, dans ces simulations, la masse volumique et la viscosité sont fonctions linéaires du taux de présence et auront donc des évolutions parallèles.

\subsection{Configuration de base}

Afin de modéliser les profils verticaux des grandeurs étudiées au voisinage de l'interface entre la vase et l'eau, nous considérons la famille des fonctions $F(\lambda, Z)$ définies par : 


$$
F(\lambda, Z)= \begin{cases}\lambda\left[1+\operatorname{erf}\left(\frac{Z}{\lambda}\right)\right] & \text { si } \quad Z \leq 0, \\ 1-(1-\lambda)\left[1-\operatorname{erf}\left(\frac{Z}{1-\lambda}\right)\right] & \text { si } \quad Z \geq 0,\end{cases}
$$

où le paramètre $\lambda$ contrôle l'asymétrie des domaines situés de part et d'autre du point d'inflexion, avec continuité du profil et de sa dérivée autour de ce point. La fonction $\operatorname{erf}(z)$, solution exacte des problèmes de diffusion moléculaire a été choisie comme fonction de base. Les caractéristiques de cette famille de profils sont décrites par la figure 1.

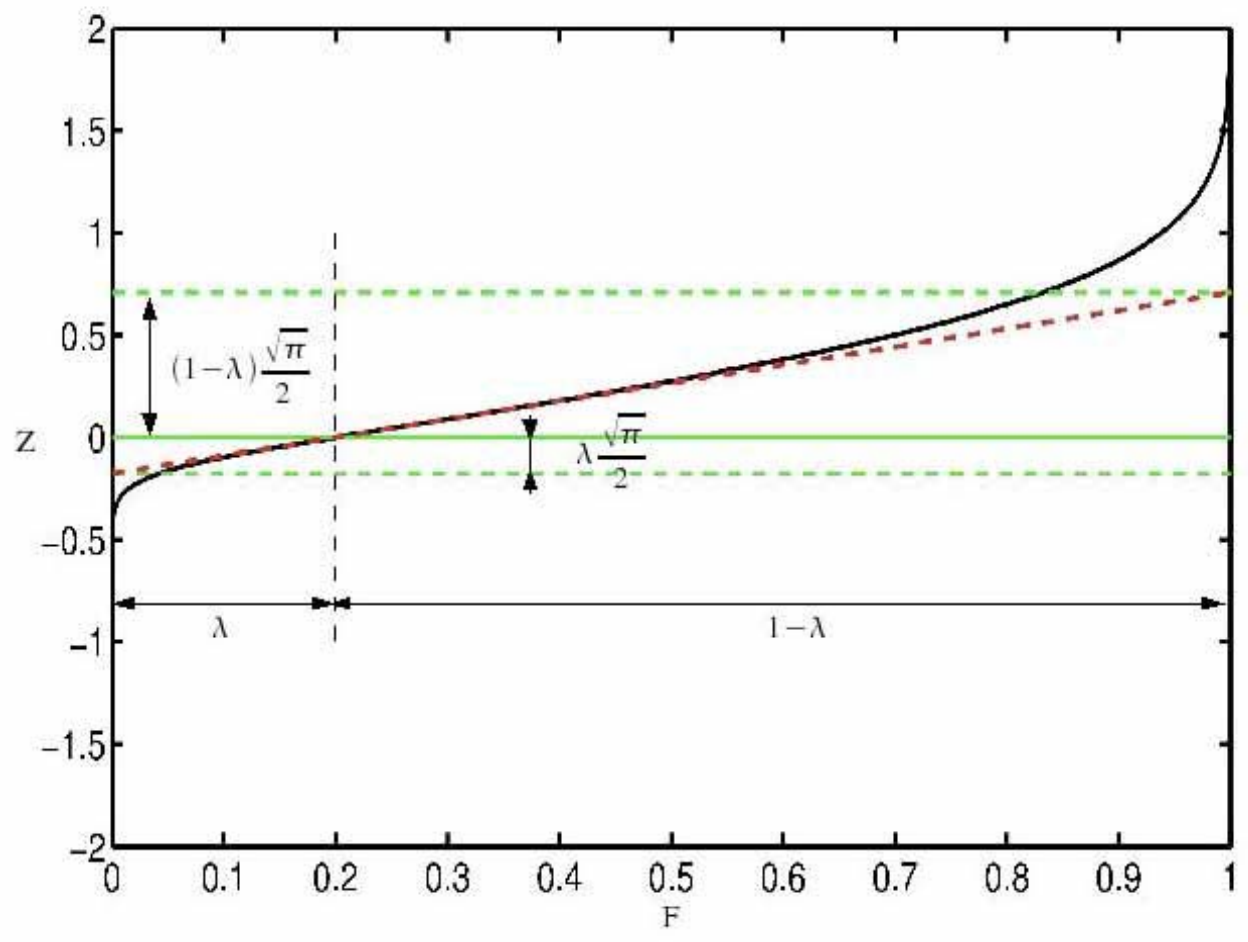

Figure 1. Représentation du profil en $Z$ de la fonction $F(\lambda, Z)$ avec $\lambda=0.2$.

On considère alors les profils de vitesse horizontale $u(z)$, de masse volumique $\rho(z)$ et de viscosité dynamique $\mu(z)$ donnés par les relations

$$
\begin{gathered}
u(z)=U_{1} F\left(\lambda, \frac{z-h}{\delta_{u}}\right), \\
\rho(z)=\left(\rho_{0}-\rho_{1}\right) F\left(\lambda, \frac{z-h}{\delta_{\rho}}\right)+\rho_{1}, \quad \mu(z)=\left(\mu_{0}-\mu_{1}\right) F\left(\lambda, \frac{z-h}{\delta_{\mu}}\right)+\mu_{1},
\end{gathered}
$$

où $\delta_{u}, \delta_{\rho}$ et $\delta_{\mu}$ sont des épaisseurs respectivement associées aux profils $u(z), \rho(z)$ et $\mu(z)$. Par définition des variables, $\delta_{\rho}$ et $\delta_{\mu}$ sont égales, par soucis de simplification, une même 
valeur est choisie pour $\delta_{u}: \delta=0.3 \mathrm{~m}$, correspondant à l'épaisseur de la couche de mélange. Les frontières du domaine de la simulation étant définies par les équations $z=0$ et $z=3 h$, le choix d'une hauteur $h=1 \mathrm{~m}$ permet de les situer suffisamment loin de l'interface d'équation $z=h$. D'autre part, nous avons choisi $\lambda=0.1$, en considérant que la diffusion est plus importante dans l'eau que dans la vase. Nous obtenons alors les profils présentés sur la figure 2 .
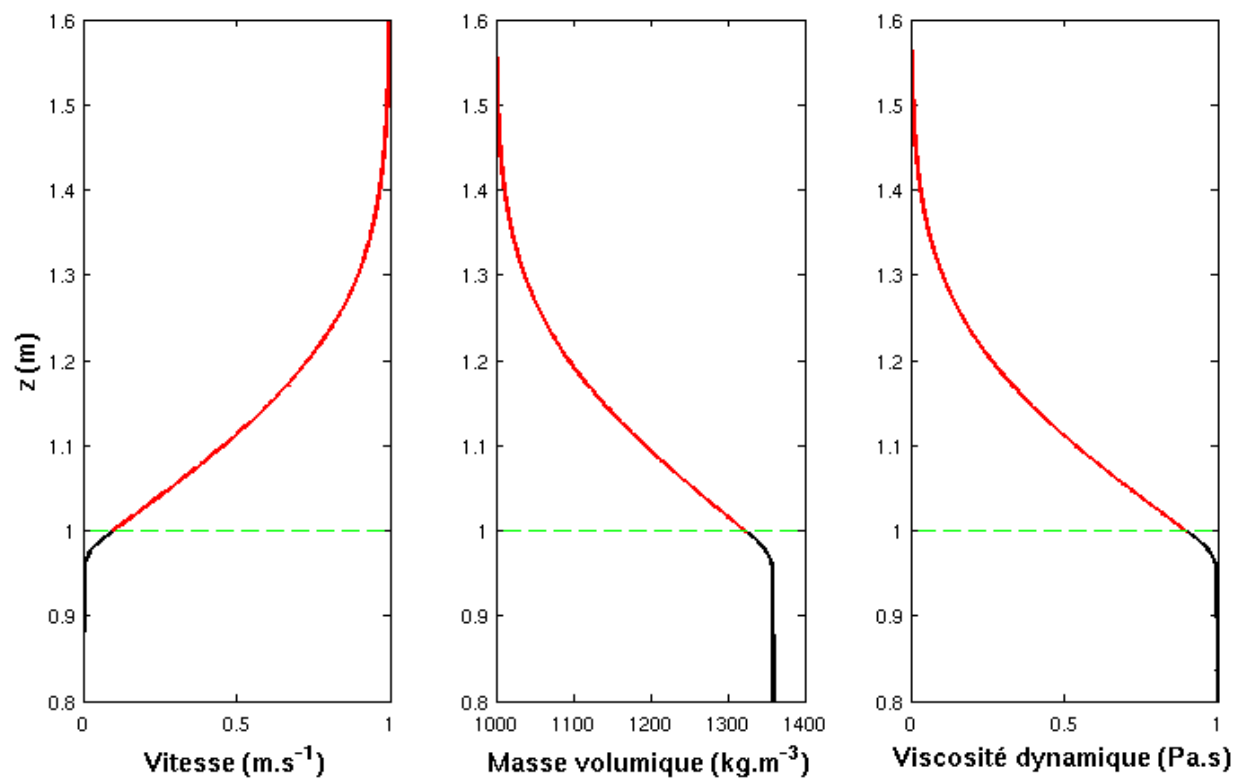

Figure 2. Profils initiaux $u(z), \rho(z)$ et $\mu(z)$ sur une portion $z \in[0.8 \mathrm{~m}, 1.6 \mathrm{~m}]$ du domaine $z \in[0,3 \mathrm{~h}]$, avec $h=1 \mathrm{~m}, \delta=0.3 \mathrm{~m}$ et $\lambda=0.1$.

À l'instant initial, l'écoulement parallèle est perturbé par un champ de vitesse verticale localisé autour de l'interface $z=h$ et composé de dix harmoniques en $x$ d'amplitude $w_{m}=10^{-3} \mathrm{~m} \mathrm{~s}^{-1}$. La simulation est alors effectuée dans un domaine bidimensionnel périodique $x$ de période $L=3 \mathrm{~m}$ avec une condition de condition d'adhérence en $z=0$ et de glissement en $z=3 h$. La taille des mailles dans ce domaine carré est de $1 \mathrm{~cm}$, la résolution étant alors de $300 \times 300$.

\subsection{Paramètres de contrôle}

Nous nous plaçons tout d'abord dans des configurations où le nombre d'Atwood $\left(\rho_{1}-\rho_{0}\right) /\left(\rho_{1}+\rho_{0}\right)$ est de l'ordre de 0.15 . Les trois autres nombres adimensionnels pertinents pour ce problème sont définis par :

$$
R e=\frac{U_{0} \delta}{\mu_{0} / \rho_{0}}, \quad R i=\frac{g}{\rho_{h}} \frac{\left(\rho_{0}-\rho_{1}\right) \delta \sqrt{\pi}}{2 U_{0}^{2}} \text { et } W=\log _{10}\left(\frac{\mu_{1}}{\mu_{0}}\right),
$$

Où $\rho_{h}=\rho(h)=\left(\rho_{0}-\rho_{1}\right) \lambda+\rho_{1}$ est la masse volumique à l'interface, le nombre de Reynolds $R e$ est basé sur la viscosité de l'eau, le nombre de Richardson $R i$ est basé sur 


\section{XI $I^{\text {èes }}$ Journées Nationales Génie Côtier - Génie Civil \\ Les Sables d'Olonne, 22-25 juin 2010}

le maximum du gradient de masse volumique situé à l'interface $z=h$, et où nous avons choisi d'exprimer le rapport des viscosités entre la vase et l'eau par son logarithme décimal $W$.

\section{Résultats}

On choisit de conserver, dans les simulations, les valeurs de masse volumique de l'eau et de la vase déterminée à partir de MEHTA et al. (1989). Nous faisons alors varier la vitesse afin de modifier le nombre de Richardson $R i$ et le nombre de Reynolds $R e$, ainsi que la viscosité de la vase pour faire varier $W$. Les caractéristiques des différentes configurations des simulations discutées ici sont présentées dans le tableau 1.

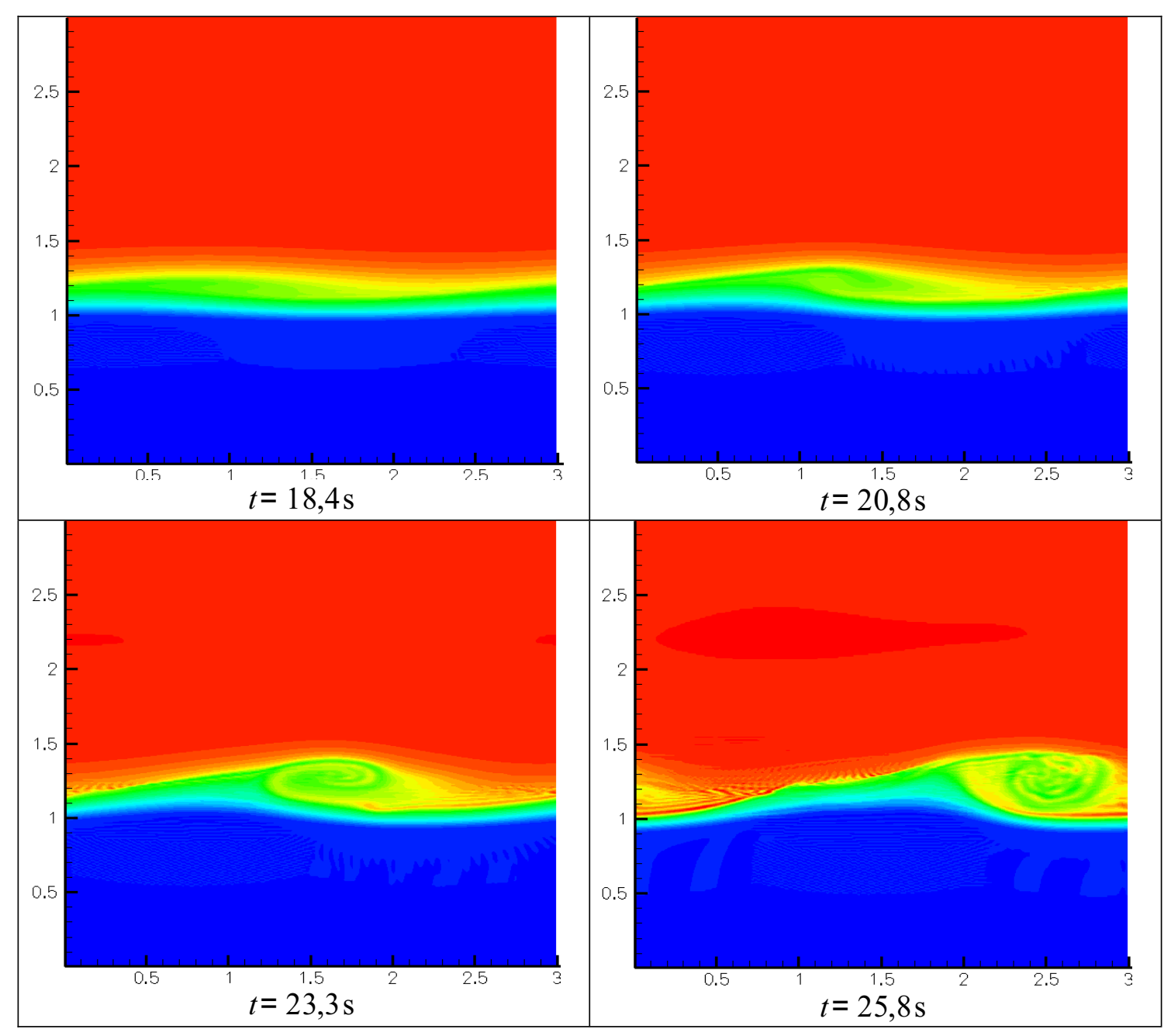

Figure 3. Configuration A.3 ( $R i=0.113$ et $W=3$ ). Champ de masse volumique $\rho(x, z) \grave{a}$ différents instants séparés de $2.5 \mathrm{~s}$. 
Thème 2 -Dynamique sédimentaire et transports des particules

Tableau 1. Présentation des configurations en fonction des paramètres des simulations.

\begin{tabular}{llll}
\hline & $R i=0.113$ & $R i=0.232$ & $R i=0.315$ \\
& $U_{0}=2.50 \mathrm{~m} \mathrm{~s}^{-1}$ & $U_{0}=1.75 \mathrm{~m} \mathrm{~s}^{-1}$ & $U_{0}=1.50 \mathrm{~m} \mathrm{~s}^{-1}$ \\
\hline$W=3$ & configuration A.3 & configuration B.3 & configuration C.3 \\
& instable (figure 3) & instable (figure 4) & stable \\
& configuration A.1 & configuration B.1 & configuration C.1 \\
& instable (figure 5) & stable & stable \\
\hline
\end{tabular}
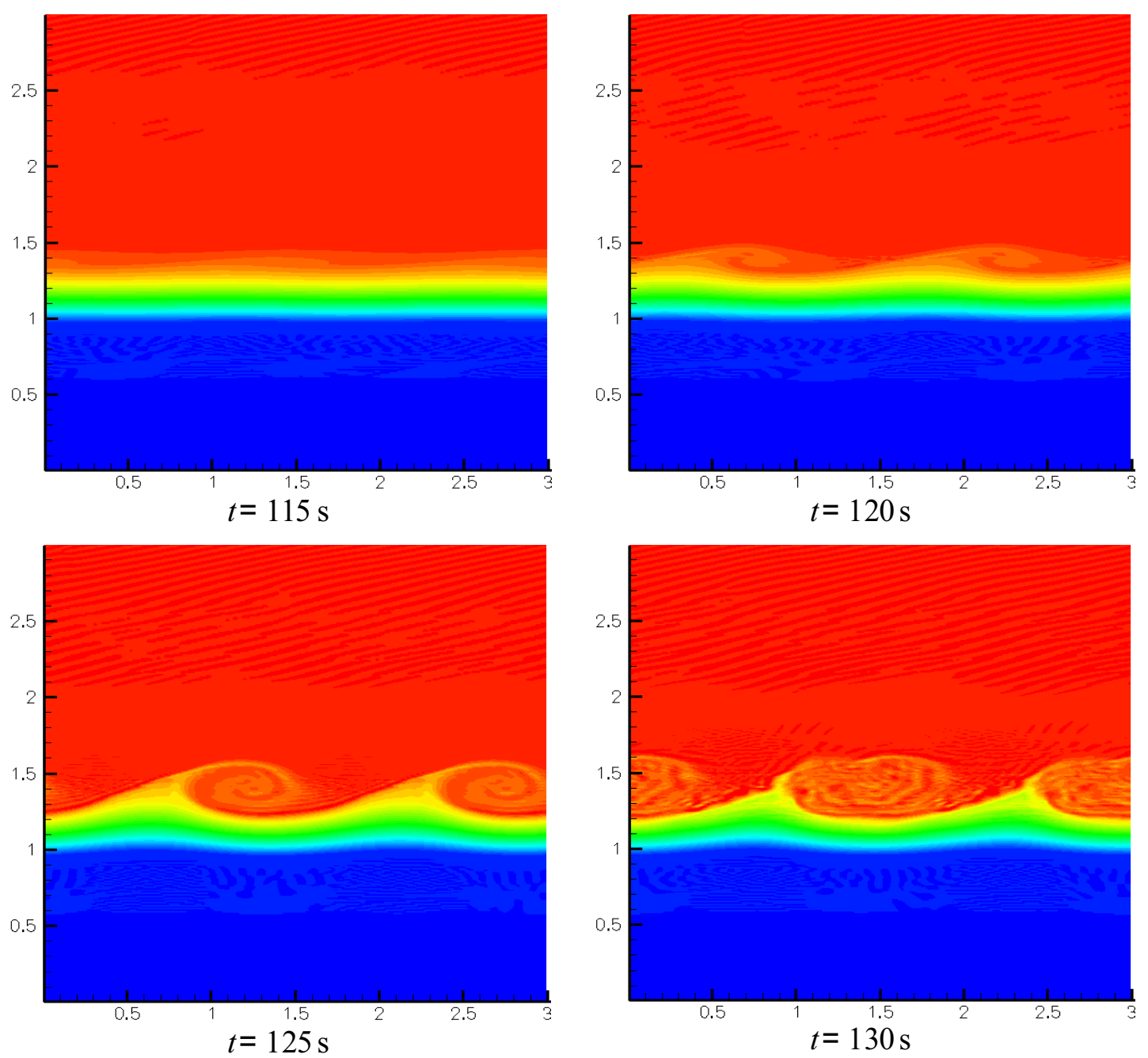

Figure 4. Configuration B.3 (Ri=0.232 et $W=3)$. Champ de $\rho(x, z)$ tous les $5 \mathrm{~s}$. 


\section{XI $I^{\text {emes }}$ Journées Nationales Génie Côtier - Génie Civil \\ Les Sables d'Olonne, 22-25 juin 2010}

\section{1 Étude de stabilité dans un cas proche de la réalité}

On s'intéresse tout d'abord aux configurations $W=3$, correspondant au cas $\mu_{1}=1 \mathrm{~Pa}$ s. L'écoulement est stable pour la configuration C.3 (non montré) : les faibles oscillations, dues à l'introduction des perturbations autour de l'interface $z=h$, s'atténuent au cours du temps. D'autre part, les configurations A.3 et B.3, présentant un nombre de Richardson inférieur ou égal à 0.24 , déclenchent une instabilité de type Kelvin-Helmholtz (voir figures 3 et 4). On observe, dans les deux cas, la formation de rouleaux, mais de longueurs caractéristiques différentes ( $3 \mathrm{~m}$ pour A. 3 et $1.5 \mathrm{~m}$ pour B.3).

Pour les cas instables, plus le nombre de Richardson est petit, plus l'interface se déstabilise vite : $115 \mathrm{~s}$ pour $R i=0.232$ (B.3) contre $18 \mathrm{~s}$ pour $R i=0.113$ (A.3).
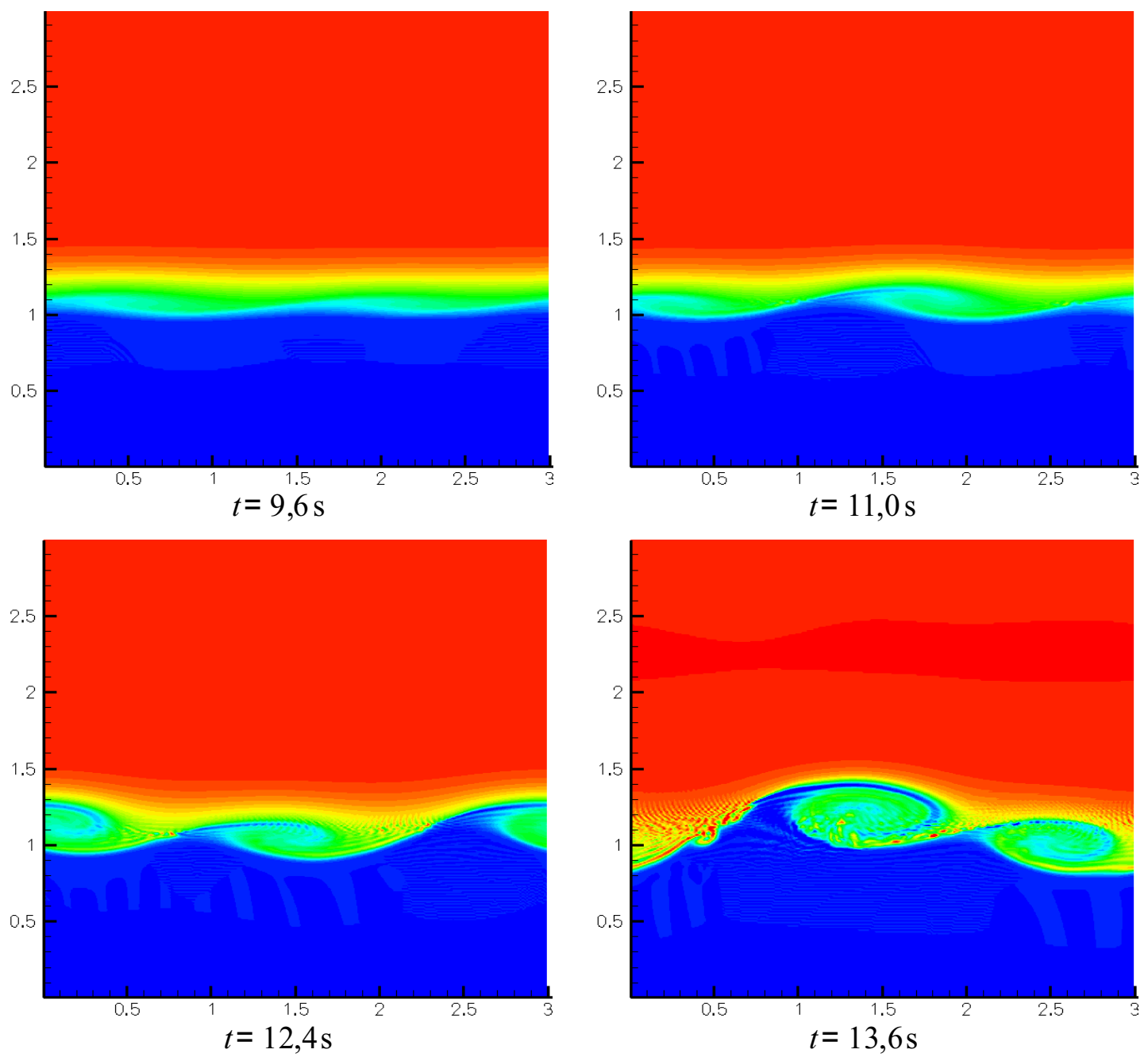

Figure 5. Configuration A.1 (Ri=0.113 et $W=1)$. Champ de $\rho(x, z)$ tous les $1.3 \mathrm{~s}$ 


\subsection{Influence du gradient de viscosité sur la stabilité}

Pour étudier maintenant l'effet du gradient de viscosité sur la stabilité de cette interface de type eau/crème de vase, on diminue la viscosité de la vase à $\mu_{1}=0.001 \mathrm{~Pa}$ s soit $W=1$. On constate tout d'abord que, contrairement à la configuration B.3, la configuration B.1 est stable : proche du Richardson critique, un fort gradient de viscosité a donc un effet déstabilisant. Pour la configuration $\mathrm{A}$, instable pour $W=1$ et $W=3$, on remarque qu'un plus faible gradient de viscosité tend à accélérer la formation de rouleaux (voir figures 4 et 5).

\section{Conclusions}

Nous avons modélisé l'interface entre la crème de vase et l'eau par deux fluides newtoniens miscibles présentant de fortes variations de masse volumique et de viscosité. A l'aide de simulations numériques $2 \mathrm{D}$, nous avons observé une limite de stabilité $R i=1 / 4$ proche de la limite théorique connue pour les écoulements cisaillés stratifiés de viscosité homogène. Nos résultats montrent que le gradient de viscosité influe sur déclenchement de l'instabilité, en modifiant le nombre de Richardson critique ainsi que la longueur d'onde du mode le plus instable. Le rapport de viscosité doit donc être pris en compte dans la paramétrisation du déclenchement de mélange à l'interface par instabilité de cisaillement.

Ces résultats motivent une étude paramétrique plus complète ainsi que le passage à une configuration 3D. Il est aussi prévu de prolonger ce travail en introduisant une rhéologie propre à la vase.

\section{Remerciements}

Les auteurs remercient Thomas BONOMETTI pour son expertise sur le code JADIM.

Ce travail bénéficie du soutien financier de la Délégation Générale de DCA l'Armement (DGA) à travers une bourse de thèse attribuée à l'un des auteurs.

\section{Références bibliographiques}

CAULFIELD C.P., PELTIER W.R. (2000). The anatomy of the mixing transition in homogeneous and stratified free shear layers. Journal of Fluid Mechanics, $\mathrm{n}^{\circ} 413$, pp 1-47. doi:10.1017/S0022112000008284

DEARDORFF J.W., WILLIS G.E. (1982). Dependence of mixed-layer entrainment on shear stress and velocity jump. Journal of Fluid Mechanics, $\mathrm{n}^{\circ} 115$, pp 123-150. doi:10.1017/S0022112082000688

ERN P., CHARRU F., LUCHINI P. (2003). Stability analysis of a shear flow with strongly stratified viscosity. Journal of Fluid Mechanics, $\mathrm{n}^{\circ} 496$, pp 295-312. doi:10.1017/S0022112003006372 


\section{XI $I^{\text {èes }}$ Journées Nationales Génie Côtier - Génie Civil}

Les Sables d'Olonne, 22-25 juin 2010

HALLEZ Y. (2007). Mélange gravitationnel de fluides en géométrie confinée. Thèse, Institut National Polytechnique de Toulouse, 140 p.

[URL http://oa.imft.fr/1851/1/hallez2007.pdf]

KRANENBURG C., WINTERWERP J.C. (1997). Erosion of fluid mud layers. I: Entrainment model. Journal of Hydraulic Engineering, vol. 123, ${ }^{\circ} 6$, pp 504-511. doi:10.1061/(ASCE)0733-9429(1997)123:6(504)

LEGENDRE D. (1996). Quelques aspects des forces hydrodynamiques et des transferts de chaleur sur une bulle sphérique. Thèse, Institut National Polytechnique de Toulouse, 266 p. [URL http://ethesis.inp-toulouse.fr/archive/00000655/\%5D]

LE NORMAND C. (1995). Modélisation numérique tridimensionnelle des processus de transport des sédiments cohésifs en environnement estuarien. Thèse, Institut National Polytechnique de Toulouse, $237 \mathrm{p}$.

MEHTA A.J., HAYTER E.J., PARKER W.R., KRONE R.B., TEETER A.M. (1989). Cohesive sediments transport. Journal of Hydraulic Engineering, vol. 115, $\mathrm{n}^{\circ} 8$, pp 1076-1112. doi:10.1061/(ASCE)0733-9429(1989)115:8(1076) 
Thème 2 -Dynamique sédimentaire et transports des particules

\section{Annexe : Notations}

$A_{0} \quad$ : grandeur A relative à l'eau

$t \quad:$ temps (s)

$A_{1} \quad$ : grandeur A relative à la crème de $\quad \underline{u} \quad$ : vecteur vitesse $\left(\mathrm{m} \mathrm{s}^{-1}\right)$ vase

C : fraction volumique de crème de vase dans le fluide

$F \quad$ : fonction

$w_{m} \quad$ : amplitude des perturbations de vitesse verticale $\left(\mathrm{m} \mathrm{s}^{-1}\right)$

$W \quad$ : logarithme décimal du rapport de viscosité

G : accélération de la pesanteur $\left(\mathrm{m} \mathrm{s}^{-2}\right)$

$x, z \quad$ : coordonnées horizontale et verticale $(\mathrm{m})$

$H \quad$ : hauteur de l'interface eau - crème de vase $(\mathrm{m})$

: coordonnée verticale

(ici $Z=(z-h) / \delta$ )

$L \quad$ : longueur de la boite de simulation $\delta \quad$ : épaisseur de l'interface (m) (m)

$P \quad:$ pression $(\mathrm{Pa})$

$\lambda \quad$ : rapport de forme entre crème de

Re : nombre de Reynolds vase et eau

$\mu \quad$ : viscosité dynamique (Pa s)

$R i \quad$ : nombre de Richardson gradient $\quad \rho \quad$ : masse volumique $\left(\mathrm{kg} \mathrm{m}^{-3}\right)$ 